\title{
The Improvement of Oil-water Separation Technology in Oil Spill Mechanical Recovery
}

\author{
Zhang Yindong \\ College of Marine Engineering \\ Dalian Maritime University \\ Dalian, China \\ dong-zyd@163.com \\ Zhang Xingming \\ College of Marine Engineering \\ Dalian Maritime University \\ Dalian, China \\ Zhangxingming1985@163.com
}

\author{
Yang Jie \\ College of Marine Engineering \\ Dalian Maritime University \\ Dalian, China \\ yangjie@163.com \\ Li Wenhua \\ College of Marine Engineering \\ Dalian Maritime University \\ Dalian, China \\ Liwenhua992@sina.com
}

\begin{abstract}
The new methods of negative pressure suction and curved surface diversion are presented to improve oil-water separation in oil spill mechanical recovery. With the negative pressure suction, the oil-water mixture recovered by skimmer is inhaled into separator smoothly and bigger oil droplet is obtained. With curved surface diversion, the oil droplets in the separator obtain a base upward velocity in the flow field and rapid separation is achieved. By the simulation and analysis of separation process, the density and viscosity of oil droplet have small influence on the separation speed, so the methods of negative pressure suction and curved surface diversion can be utilized to fulfill oil-water rapid separation in oil spill mechanical recovery.
\end{abstract}

Keywords-oil spill; mechanical recovery; skimmer; oilwater separation

\section{INTRODUCTION}

Because of tanker accidents, vessel collisions, industrial and urban discharges, or offshore exploration and production, oil spill has become a major issue in the protection of the marine environment over the past decades. The oil spill response is to minimize the overall impacts on natural and economic resources with all possible technical means. Mechanical recovery is the most commonly used oil spill response technology which can recover floating oil from the water surface using mechanized equipment known as skimmer. In the process of cleaning up, the oil ratio of oil-water mixture recovered is an important parameter to determine the capacity and effectiveness of the mechanical recovery system because low oil content decrease actual amount of pure oil recovery and effective storage capacity of recovered material. Therefore, the requirements of oil-water separation technology in marine oil spill recovery, which includes large capacity, high separation speed and good adaptability to oil characteristics, are urgent to be met at present $[1,2,3]$.

\section{THE OIL-WATER SEPARATION PROCESS}

Due to density difference between oil and water, the working principle of the gravity type oil water separation is that buoyancy force produced by the density difference is used to achieve oil water separation. The main factors determining performance of gravity separation are droplet size and density difference. Moreover, Viscosity also has important impact on separation effectiveness [4,5]. Separation is governed by Stokes Law. This law assumes laminar flow, spherical droplets and ideal droplet distribution. Although these conditions are difficult to achieve for separation of recovered oil, Stokes Law can be used to illustrate the effect of changes in oil properties on separation of both oil and emulsion droplets in water. The two forces acting on a droplet of oil in water are the buoyancy and drag forces. The oil droplet in water is forced by buoyancy to move upward at a velocity which is related to the density differential between oil and seawater. The drag force, which is a function of velocity, opposes the buoyancy force and the rise velocity reaches a terminal value when the two forces are equal. Terminal velocity $\left(V_{t}\right)$, is used as a separator design criteria, and determines droplet size regimes that can be separated at a given resident time and throughput capacity. The buoyancy force $F_{B}$ is given by $(\pi / 6) d^{3}\left(\rho_{w}-\rho_{o}\right) g$ and the drag force (in Stokes) $F_{D}$ is $3 \pi \mu V d$. The net separation force is the difference between the two. The terminal velocity $V_{t}$ is reached when buoyancy force equals drag force and shown as follows $[6,7]$.

$$
V_{t}=\frac{d^{2}\left(S_{w}-S_{o}\right) g}{18 \mu}
$$

The $\mu$ is the kinematic viscosity of the continuous phase of the fluid. For separation of oil droplets, the continuous phase is sea water. $\left(\rho_{w}-\rho_{o}\right)$ is the difference in density between seawater and oil, $d$ is the droplet diameter and, $g$ is the gravity force. The effectiveness of separation can theoretically be improved by increasing the buoyancy 
force and/or droplet diameter, or replacement of gravitational force by centrifugal force, and by lowering the viscosity [8].

\section{THE IMPROVEMENT OF OIL-WATER SEPARATION IN OIL SPILL MECHANICAL RECOVERY}

\section{A. A New Rapid Separation Method}

The new separation methods of negative pressure suction and curved surface diversion are presented as follows.

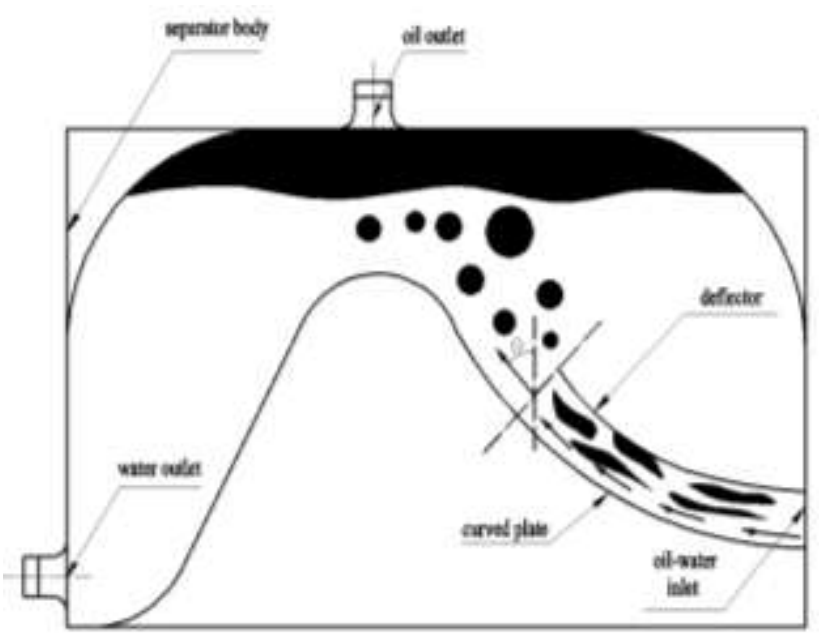

Figure 1. The schematic of new oil-water separator

The device shown in Figure 1 is a separator which is used to achieve oil-water rapid separation in oil spill recovery. The separator body is a sealed box with one inlet and two outlets, and the inlet is connected with outer skimmer. One outlet is connected with water pump and the other is connected with oil pump. Inside the separator body, there is a curved channel which is composed of curved surfaces. While working, the separator is filled with water and the water pump is started, then the water in separator is discharged by water pump and the negative pressure shall be produced, hence, the oil-water mixture recovered by skimmer shall be inhaled into the separator curved channel through inlet. At the end of curved channel, the oil-water separation occurs because of the oil-water density difference, and under the diversion effect of curved surfaces, oil droplets obtain upward velocity and float to accumulate at the top of separator. Since the rising droplets in the separator are subjected to composite force of upward flow and buoyancy, the oil-water separation velocity is increased significantly. When the oil in the separator reaches a certain thickness, the oil pump is started to transport gathered oil to the designated location [9].

\section{B. The Kinetic Analysis of Separation Process}

Under normal circumstances, the forces acting on a single oil droplet when sinking or rising in the static fluid are viscous resistance $F_{D}$, buoyancy $F_{B}$ and gravity $F_{G}$, the kinetic equation of oil droplet in the gravitational field can be obtained and shown as follows.

$$
m \frac{d u}{d t}=F_{D}+F_{B}+F_{G}
$$

To the spherical droplet, if just considering rising movement along the vertical direction, the above formula can be modified as the following [10].

$$
\frac{d u}{d t}=\frac{g\left(\rho_{w}-\rho_{o}\right)}{\rho_{o}}-C_{D} \frac{3 \rho_{w} u^{2}}{\rho_{o} \delta}
$$

The $C_{D}$ is drag coefficient determined by Reynolds number of oil droplet, $\delta$ is oil droplets size, $\mu$ is the dynamic viscosity of sea water, and $\boldsymbol{u}$ is relative velocity along the vertical direction between oil droplet and sea water.

As shown in Figure 1, the angle between the normal direction of the flow channel end and the vertical direction is $\theta$ which determines flow direction in the separator. Assuming the average upward velocity of oil-water mixture through the curved channel is given by $U_{c}$, the upward base velocity $U_{b}$ of oil droplet in the separator can be given by $U_{c} \cos \theta$.

According to the above formula, when the initial condition is that $u_{y}=0$ when $t=0$, the rising velocity of oil droplet can be obtained as follows.

$$
u_{y}=\frac{1}{A}\left(F_{B}-e^{\ln F_{b}-\frac{A}{M_{d}} t}\right)
$$

In the above formula, $\mathrm{A}=3 \pi \mu \delta, F_{B}=\frac{\pi}{6} \delta^{3}\left(\rho_{F}-\rho_{p}\right) g$, $M_{d}=\frac{\pi \delta^{3}}{6} \rho_{p}$

If $u=\frac{d y}{d t} \quad, \quad$ and the initial condition is that $y=0$ when $t=0$, the function relation between oil droplet rising height and time in the absence of base velocity is achieved as follows.

$$
y=\frac{F_{B} t}{A}+\frac{F_{B} M_{d}}{A^{2}}\left(e^{-\frac{A}{M_{d}} t}-1\right)
$$

If considering the upward basic velocity $U_{b}$ of oil droplet, the overall upward velocity of oil droplet in the separator is given as follows.

$$
u_{y}=\frac{1}{A}\left(F_{B}-e^{\ln F_{b}-\frac{A}{M_{d}} t}\right)+U_{b}
$$

According to the above formula, if $u=\frac{d y}{d t}$ and the initial condition is that $y=0$ when $t=0$, the function relation between the rising height of oil droplet with base velocity and time is achieved as follows.

$$
y=U_{b} \cdot t+\frac{F_{B} t}{A}+\frac{F_{B} M_{d}}{A^{2}}\left(e^{-\frac{A}{M_{d}} t}-1\right)
$$




\section{The Simulation Analysis of Separation Process}

If the angle between the normal direction at the end of flow channel and the vertical direction is $\theta=60^{\circ}$ and the flux of water pump is adjustable, the upward base velocity of oil droplet can be $0 \mathrm{~m} / \mathrm{s} 、 0.1 \mathrm{~m} / \mathrm{s} 、 0.2 \mathrm{~m} / \mathrm{s} 、 0.3 \mathrm{~m} / \mathrm{s}$ respectively. When oil droplet sizes are respectively $1 \mathrm{~mm}$, $2 \mathrm{~mm}$, the density of oil droplet $\rho_{o}$ is $0.80 \times 10^{3}$, the density of seawater $\rho_{w}$ is $1.03 \times 10^{3}$, the dynamic viscosity $\mu$ is $1 \times 10^{-3} \mathrm{~Pa} \llbracket \mathrm{s}$, the relation between time and rising height of oil droplet in the separator is achieved and shown as follows.

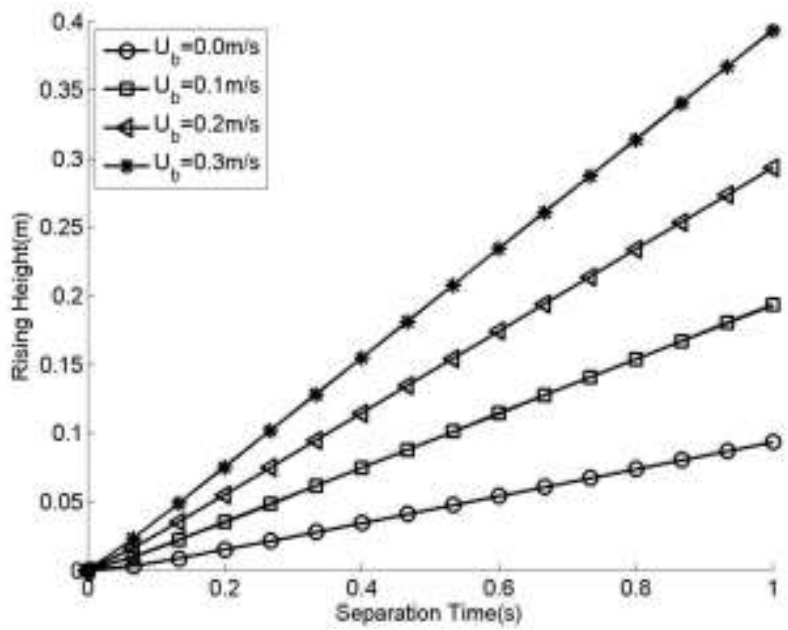

Figure 2. The relation between time and the rising height of oil droplets with different base velocity ( $\delta=0.5 \mathrm{~mm}$ )

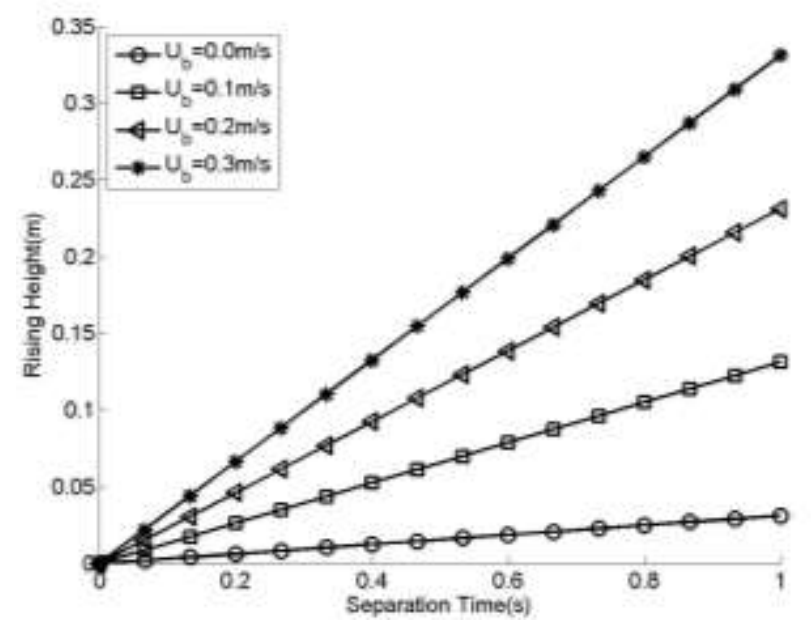

Figure 3. The relation between time and the rising height of oil droplets with different base velocity ( $\delta=2.0 \mathrm{~mm}$ )

As is shown by the Figure 2 and 3 above, the oil droplets with upward base velocity can easily float to the top of separator and fulfill oil-water separation with higher speed, to small oil droplets, the improvement effect of separation are more obvious.

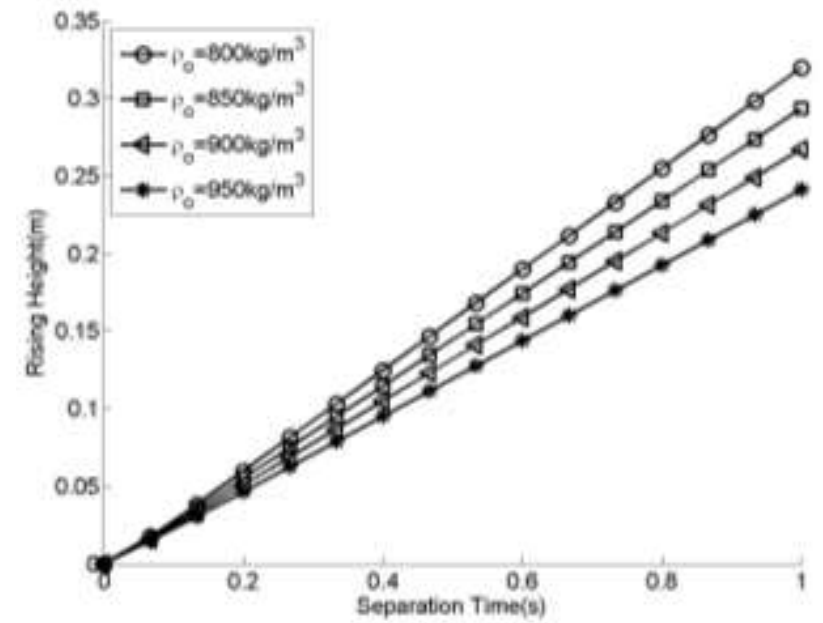

Figure 4. The relation between time and the rising height of oil droplets with different density

If the assumptions are $U_{b}=0.2 \mathrm{~m} / \mathrm{s}, \quad \delta=1 \mathrm{~mm}$, $\mu=1 \times 10^{-3} \mathrm{~Pa}\left\lceil\mathrm{~s}\right.$ and the oil droplet density values $\rho_{o}$ are respectively $800 \mathrm{~kg} / \mathrm{m} 3,850 \mathrm{~kg} / \mathrm{m} 3,900 \mathrm{~kg} / \mathrm{m} 3$, $950 \mathrm{~kg} / \mathrm{m} 3$, the relation between time and rising height of oil droplet with different density in the separator is achieved and shown by figure 3 .

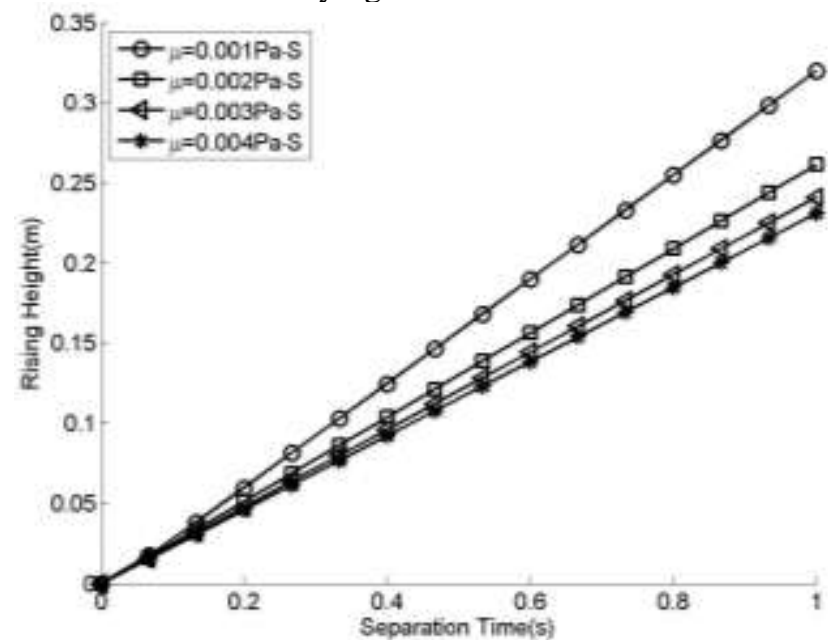

Figure 5.The relation between time and the rising height of oil droplets with different viscosity

As is shown by the Figure 4 and 5 above, with the same oil droplet size and upward base velocity, the density and viscosity of oil droplet have small influence on the separation speed. So the separation method with negative pressure suction and curved surface diversion can be utilized to fulfill rapid separation of small emulsified oil droplet with high density and viscosity.

\section{CONCLUSIONS}

The new methods of negative pressure suction and curved surface diversion are presented to improve oilwater separation effect. By the method, the oil-water mixture recovered by skimmer can be inhaled into separator smoothly and obtain an upward base velocity in the flow field, so the oil droplets could obtain larger diameter and float upward with higher speed. 
The kinetic analysis of oil-water separation process is carried out, and according to the simulation analysis of separation process with the new methods, it can be found that the separation effect of smaller oil droplet is improved significantly and the density and viscosity of oil droplet have small influence on the separation speed, so the new method thus can satisfy the requirements of large capacity, high separation speed and good adaptability to oil characteristics and even be employed to fulfill rapid separation of emulsified oil in oil spill mechanical recovery.

\section{ACKNOWLEDGMENT}

The authors are very grateful to the financial support from the Fundamental Research Funds for the Central Universities (No.3132013028) and National Natural Science Foundation Item (No. 51109021).

\section{REFERENCES}

[1] Ornitz, Barbara E., Esq. Champ, Michael A., "Oil Spill First Principles: Prevention \& Best Response", Elsevier, Amsterdam, 2002.

[2] Atle B.Nordvik, "Time window-of-opportunity strategies for oil spill planning and response", Pure Appl. Chem., vol.71 (1), 5-16, 1999.

[3] Owens, E. H., "Practical Guidelines or 'Rules of Thumb' for Spill Response Activities," Proceedings of the Twenty-Second Arctic and Marine Oil spill Program (AMOP) Technical Seminar, June 2 to 4, 1999, Calgary, Alberta, Canada, Environment Canada, pp. 695-704.

[4] Bitting, K. R., Nordvik, A. B. and Murdoch, M. A., "Tests of oil/water separators for spilled oil recovery operations", In Proceedings of the Marine Technology Society Conference 1993, Washington DC, pp. 222-228.

[5] Benxin Jing, Haitao Wang, Kun-Yi Lin, Paul J. McGinn, Chongzheng $\mathrm{Na}$, Yingxi Zhu, A facile method to functionalize engineering solid membrane supports for rapid and efficient oilwater separation, Polymer, Vol. 54, Issue 21, 4 October 2013, pp. 5771-5778

[6] Chen Jianlei, He Limin, Luo Xiaoming, Bai Haitao, Wei Yanhai. Simulation of oil-water two phase flow and separation behaviors in combined T junctions, Journal of Hydrodynamics, Vol. 24, Issue 6 , December 2012, pp. 848-857.

[7] Nikolaos P. Ventikos, Emmanouil Vergetis, Harilaos N.Psaraftis, George Triantafyllou, "A high-level synthesis of oil spill response equipment and countermeasures", Journal of Hazardous Materials, vol.107, 51-58,2004.

[8] Atle B. Nordvik, James L. Simmons, Kenneth R. Bitting, "Oil and water separation in marine oil spill clean-up operations", Spill Science \& Technology Bulletin. vol.3 (3), 107-122,1996.

[9] Zhang Yindong, Sun Yuqing, Hou Jiemin, Li Wenhua, “Analysis of increasing velocity of oil-water separation by induction of negative pressure", Journal of China University of Petroleum. vol.31 (2), 114-117, 2006.

[10] Lu Yaojun, Xue Dunsong. A mathematical model for gravity oi water separation. Journal of Petroleum (Petroleum Processing), 15(3): 34-41, 1999. 